\title{
Change in relaxation scenario at the order-disorder transition of a colloidal fluid of hard spheres seen from the Gaussian limit of the self-intermediate scattering function
}

Van Megen, William; Mortensen, Timothy; Bryant, Gary

https://researchrepository.rmit.edu.au/esploro/outputs/9921858479101341/filesAndLinks?institution=61RMIT_INST\&index=null

Van Megen, W., Mortensen, T., \& Bryant, G. (2005). Change in relaxation scenario at the order-disorder transition of a colloidal fluid of hard spheres seen from the Gaussian limit of the self-intermediate scattering function. Physical Review E - Statistical, Nonlinear, and Soft Matter Physics, 72(3), 1-9. https://doi.org/10.1103/PhysRevE.72.031402

Published Version: https://doi.org/10.1103/PhysRevE.72.031402 


\title{
Change in relaxation scenario at the order-disorder transition of a colloidal fluid of hard spheres seen from the Gaussian limit of the self-intermediate scattering function
}

\author{
W. van Megen, T. C. Mortensen, and G. Bryant \\ Department of Applied Physics, Royal Melbourne Institute of Technology, Melbourne, Victoria 3000, Australia
}

(Received 2 May 2005; published 2 September 2005)

\begin{abstract}
Self-intermediate scattering functions (ISFs) are measured by dynamic light scattering for the colloidal fluid of hard spheres for both equilibrium and nonequilibrium (undercooled) conditions, i.e., for volume fractions below and above the known freezing transition of the hard-sphere system. The delay time $\tau_{\mathrm{m}}$ where the mean-squared displacement, or the low wave-vector limit of the ISF, exhibits its maximum stretching is identified as a characteristic of the non-Markovian process(es) and is used to separate the ISF into fast $\left(\tau<\tau_{\mathrm{m}}\right)$ and slow $\left(\tau>\tau_{\mathrm{m}}\right)$ contributions. Each of these contributions exposes qualitative differences in the dynamics of the particles between the equilibrium and nonequilibrium colloidal fluids. These changes in the relaxation scenario signal the colloidal fluid's awareness of its traversal of the freezing volume fraction.
\end{abstract}

DOI: 10.1103/PhysRevE.72.031402

PACS number(s): 82.70.Dd, 64.70.Dv, 61.20.Ne

\section{INTRODUCTION}

Most liquids submit to undercooling to some degree before they solidify, usually very quickly, into a crystalline solid. In some cases it is possible to undercool the liquid to an extent where its resistance to flow becomes so great that it behaves like a brittle, but amorphous, solid, i.e., a glass is formed. Both "solidification" processes are of practical and fundamental interest and there continues to be considerable inquiry into the underlying mechanisms [1].

One line of inquiry employs suspensions of solid, nearmicrometer sized particles suspended in liquid. Interactions among the particles can be made to vary considerably and, consequently, suspensions can display a rich variety of phases [2]. Here we consider the simple case where the particles interact like hard spheres: the volume fractions of coexisting fluid and crystal phases, $\phi_{\mathrm{f}}=0.494$ and $\phi_{\mathrm{m}} \approx 0.54$, observed for the laboratory prepared "hard-sphere" suspension [3] map approximately onto those known for the perfect hard-sphere system [4]. However, unlike undercooled fluids of simple molecules, the undercooled colloidal melt of hard spheres crystallizes slowly. Although the hard-sphere system is athermal, for convenience we will still describe the colloidal fluid as "undercooled" once the volume fraction exceeds the freezing value. The large radii and damped motions of the suspended particles cause structural relaxation to be slow enough to be measured with real time correlators. In addition, a small spread in the particle radii delays formation of Bragg reflecting crystals thereby extending the lifetime of the undercooled colloidal fluid and allowing exploration of its structure and dynamical properties [5].

Experiments to date show that as the volume fraction of the colloidal fluid is increased the decay of the time correlation function of number density fluctuations becomes slower, deviates more and more from an exponential, and develops a two stepped decline to the noise floor [6,7]. The slower, second step effectively arrests at $\phi_{\mathrm{g}} \approx 0.565$, the point identified as the glass transition (GT).

The dynamic slowing just mentioned and the GT observed in glass forming fluids generally is commonly consid- ered in terms of the cage effect [8]. The microscopic visualization this conveys is that, in time, the atoms of a liquid (or, for that matter, particles in suspension) are able to diffuse arbitrarily large distances although their progress is continually hindered by temporary entrapments in instantaneous cages of neighboring atoms (or particles). The average entrapment period lengthens on increasing the liquid's density until, at the GT, all atoms are permanently trapped by their neighbors. The entrapment period represents a crossover from (local) solidity to fluidity. In this the cage picture presents a microscopic underpinning of Maxwell's model of viscoelasticity [9]. The cage picture is augmented in modecoupling theory (MCT) of the GT [10] by a delayed, nonlinear feedback that, in effect, models the cooperation or backflow required for an atom to "escape" from its neighbor cage. In essence this feedback drives the deviations of the time correlation functions from simple exponential functions of delay time. Significantly, MCT quantitatively describes the stretching, the two-stepped decay, and arrest of the density correlation function, for example, as observed not only for the colloidal fluid of hard spheres $[6,11]$ but a variety of other glass forming fluids [12].

In the preceding theories the time correlation functions are predicted to change continuously and smoothly, just like transport coefficients, as the fluid is cooled or compressed through its freezing point. In fact there is no mechanism by which the fluid is aware of its traversal through this point. Put another way, from the cage perspective the fluid does not know whether it is in thermodynamic equilibrium or whether it is undercooled. Moreover, observations to date appear to be entirely consistent with this perspective. Despite this, we still wonder whether there could be qualitative changes in the time correlation functions that signal the fluid's awareness of its passage through the freezing point.

Our purpose here is to examine, more closely than in previous work [7], the autocorrelation function of tagged particle density fluctuations to see whether there are features that may have been overlooked. We restrict ourselves to the Gaussian, or small wave-vector, limit of the self-intermediate 
scattering function (ISF). As found previously [7], neither the ISF itself nor the mean-squared displacement show any qualitative change when the volume fraction passes through the phase boundaries at $\phi_{\mathrm{f}}$ and $\phi_{\mathrm{m}}$. However, in this paper analysis of dynamic light scattering (DLS) data is taken further by adopting two methods to distinguish fast and slow contributions to the ISF. The first separates the ISF into a fast Markovian process, characterized by the short-time selfdiffusion coefficient, and a slower non-Markovian process that is indicative of structural relaxation. Obviously, this approach assumes the fastest detectable movements of the particles to be Markovian. No assumption is made in the second method where fast and slow contributions are delineated by the delay time where the ISF displays its greatest stretching.

An abridged version of the results has already been reported [13]. This paper presents a more detailed analysis and discussion. It is set out as follows: Secs. II and III, respectively, summarize dynamic light scattering theory and experimental procedures. Experimental results and their analysis are contained in Sec. IV. The summary and conclusions are presented in Sec. V.

\section{THEORY}

Dynamic light scattering, now a standard method for measuring dynamical properties of liquids, polymer solutions, and suspensions, has been reviewed in numerous articles [14]. In this section we present those aspects of the theory required for the discussion of the data.

The property of interest is the self-intermediate scattering function (ISF) or moment generating function of the particle displacement, $\Delta \mathbf{r}(\tau)$, in the time interval $\tau$,

$$
F_{\mathrm{s}}(q, \tau)=\langle\exp [i \mathbf{q} \cdot \Delta \mathbf{r}(\tau)]\rangle,
$$

where $\mathbf{q}$ is the wave vector. The procedure by which $F_{\mathrm{s}}(q, \tau)$ is obtained from the measured autocorrelation function of the intensity of the scattered light is detailed in other papers $[7,15]$.

The angular brackets in the above expression denote averages over the ensemble of the phases that appear in the exponent. The resulting rotational invariance leads to the cumulant expansion [16],

$$
\ln F_{\mathrm{s}}(q, \tau)=-\left\langle\Delta r^{2}(\tau)\right\rangle \frac{q^{2}}{6}+\frac{1}{2} \alpha(\tau)\left(\frac{q^{2}}{6}\right)^{2}+\cdots .
$$

Successive cumulants $\left\langle\Delta r^{2}(\tau)\right\rangle$ and $\alpha(\tau)=\frac{3}{5}\left\langle\Delta r^{4}(\tau)\right\rangle$ $-\left\langle\Delta r^{2}(\tau)\right\rangle^{2}$ of the particle displacement distribution, respectively, represent the mean-squared displacement (MSD) and the first deviation from Gaussian. From Eq. (2) it follows that

$$
F_{\mathrm{s}}(q \rightarrow 0, \tau)=F_{\mathrm{s}}^{(\mathrm{G})}(q, \tau)=\exp \left[-q^{2}\left\langle\Delta r^{2}(\tau)\right\rangle / 6\right],
$$

i.e., non-Gaussian contributions are not exposed in the limit of infinite spatial integration $(q \rightarrow 0)$.

A common approximation assumes the suspending liquid imparts no memory to particle displacements for any time interval that gives detectable fluctuations in the scattered light [17]. Thus the fastest detectable process is Markovian, i.e.,

$$
\left\langle\Delta r^{2}(\tau)\right\rangle=6 D_{\mathrm{s}} \tau .
$$

Clearly this approximation applies, if at all, for observation intervals no longer than the average interval $\tau_{\mathrm{c}}$ between particle encounters. Accordingly the constant, $D_{\mathrm{s}}$ in Eq. (4), is referred to as the short-time self-diffusion coefficient. We estimate $\tau_{\mathrm{c}}$ from the interval during which a particle of a suspension of hard spheres incurs a root-mean-squared (RMS) displacement equal to the average gap,

$$
R_{\mathrm{c}}=\left(\frac{\phi_{\mathrm{R}}}{\phi}\right)^{1 / 3}-1
$$

between the particles, expressed here in units of the particles' radius $R$. Since we are interested in only the disordered, fluid states we set $\phi_{\mathrm{R}}=0.64$, the volume fraction at random close packing where the particles are presumed to be touching.

One defines the ideal dilute suspension as that in which particles never encounter each other. Equation (4) then applies for all $\tau$ with $D_{\mathrm{s}}$ replaced by $D_{\mathrm{o}}$, the diffusion coefficient of an isolated particle in an unbounded liquid. In the units employed here, diffusion coefficients are expressed in units of $D_{\mathrm{o}}$, so that for the ideal dilute suspension $D_{\mathrm{s}}=1$. Any reduction of $D_{\mathrm{s}}$, relative to unity, reflects slowing of (thermally driven) diffusion of the particles by hydrodynamic modes in the suspending liquid that propagate instantaneously on the time scale of detectable fluctuations of the scattered light [17-19].

We separate the self-ISF into "fast" and "slow" contributions in two ways:

(i) The first employs the Markovian assumption, Eq. (4), for the fast contribution. The quantity

$$
T(q, \tau)=\exp \left[-q^{2} D_{\mathrm{s}} \tau\right]
$$

will be referred to as the "thermal mode." The difference,

$$
N(q, \tau)=F_{\mathrm{s}}(q, \tau)-T(q, \tau)
$$

exposes a slower, non-Markovian process characteristic of structural relaxation. From this perspective structural relaxation is presumed to occur entirely by accumulation of diffusive (uncorrelated) encounters among the particles: no account is taken of memory effects in the suspending liquid.

(ii) The second method introduces no assumption. It simply makes use of the observed stretching of the MSD. We define $\tau_{\mathrm{m}}$ as the delay time where the stretching is greatest. The stretching index at $\tau_{\mathrm{m}}$ is

$$
\nu=\min \left\lfloor\frac{d \log \left\langle\Delta r^{2}(\tau)\right\rangle}{d \log (\tau)}\right\rfloor .
$$

The corresponding MSD is $R_{\mathrm{m}}^{2}=\left\langle\Delta r^{2}\left(\tau_{\mathrm{m}}\right)\right\rangle$. From the amplitude

$$
A(q)=F_{\mathrm{s}}\left(q, \tau_{\mathrm{m}}\right)
$$

of the ISF at $\tau_{\mathrm{m}}$ we calculate the quantity 


$$
P(q, \tau)=\left|\frac{F_{\mathrm{s}}(q, \tau)-A(q)}{1-A(q)}\right| .
$$

We refer to $P\left(q, \tau<\tau_{\mathrm{m}}\right)$, the decay of the ISF towards $A(q)$, as the "fast" component of the fluctuations and $P\left(q, \tau>\tau_{\mathrm{m}}\right)$, the decay of the ISF away from $A(q)$, as the "slow" component. Thus $P\left(q, \tau<\tau_{\mathrm{m}}\right)$ and $P\left(q, \tau>\tau_{\mathrm{m}}\right)$ are the correlation functions of fluctuations accumulated in the intervals of delay times (' 0, , $\left.\tau_{\mathrm{m}}\right)$ and $\left(\tau_{\mathrm{m}}\right.$, " $\infty$ ") $)$, respectively. Here " 0 " and " $\infty$ " denote the lower and upper limits of the experimental resolution.

These definitions are well known from MCT of the glass transition $[10,12]$. There $\tau_{\mathrm{m}}$ is the crossover time from the fast $\beta$ process to the slow $\alpha$ process and $A(q)$ is the nonergodicity parameter. However, rather than analyzing the data in terms of MCT, the purpose in this paper is to see whether the quantities $P\left(q, \tau<\tau_{\mathrm{m}}\right)$ and $P\left(q, \tau>\tau_{\mathrm{m}}\right)$ reveal any qualitative changes when the volume fraction traverses $\phi_{\mathrm{f}}$.

In the limit $\tau \rightarrow \infty$, displacements must evolve towards Markovian, by definition, for a system in thermodynamic equilibrium, i.e.,

$$
\left\langle\Delta r^{2}(\tau \rightarrow \infty)\right\rangle=6 D_{1} \tau,
$$

where $D_{1}$ is the long-time self-diffusion coefficient.

\section{METHODS}

Dynamic light scattering procedures and the preparation and characterization of colloidal particles are described in detail in previous work [e.g., $[7,15]]$. Here we mention the most important points.

First, effective hard-sphere volume fractions $\phi$ of the samples were determined by referencing the observed freezing volume fraction, based on weight analysis, to the known freezing value, $\phi_{\mathrm{f}}=0.494$, of the perfect hard-sphere system [4]. This gives melting and GT volume fractions at $\phi_{\mathrm{m}}=0.535 \pm 0.005$ and $\phi_{\mathrm{g}}=0.565 \pm 0.005$, respectively $[3,6]$. From the approximate agreement between the observed miscibility gap, $\phi_{\mathrm{m}}-\phi_{\mathrm{f}}$, and that expected of the hard-sphere system, it has been inferred that the interaction between the particles is effectively equivalent to that between hard spheres [3]. This inference has been confirmed by direct measurement of the force between the particles' stabilizing layers [20]. For the particles considered here there is some narrowing of the miscibility gap due to polydispersity of the particles [21].

Second, volume fractions of the samples are in the range $0.16 \leqslant \phi \leqslant \phi_{\mathrm{g}}$. Measurements in undercooled samples $\left(\phi>\phi_{\mathrm{f}}\right)$ were made before any Bragg reflections were evident or, for that matter, before the static structure factors showed discernable crystal-like features. As reported previously, except when the suspension is in the glass state $(\phi$ $\geqslant \phi_{\mathrm{g}}$ ), the ISFs are independent of the commencement time of the measurements [7]. Accordingly, the measurements reported here were made over a space-time window in which the suspension's properties are (quasi)stationary in space and time.

Third, in order to measure self ISFs, tracer systems are used. The suspensions consist of polymer particles closely

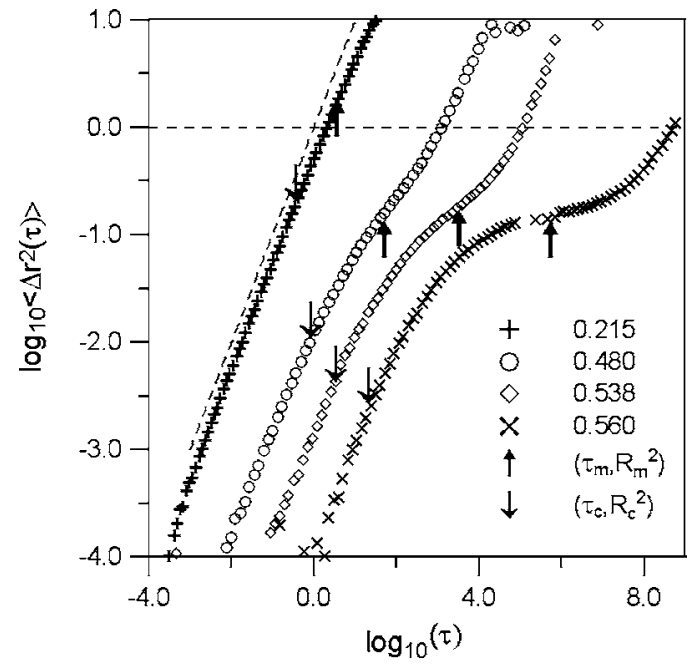

FIG. 1. Double logarithm plot of the MSD vs delay time at the volume fractions indicated. The arrows indicate the points $\left(\tau_{\mathrm{c}}, R_{\mathrm{c}}^{2}\right)$ and $\left(\tau_{\mathrm{m}}, R_{\mathrm{m}}^{2}\right)$, respectively (see text for more information). The dashed line is the MSD for the ideal dilute suspension.

indexed matched with the suspending liquid, with approximately $2 \%$ (by volume) of silica particles of the same average radius $(R=200 \mathrm{~nm})$. Both particles are sterically stabilized by the same coating of poly-12-hydroxystearic acid of thickness about $10 \mathrm{~nm}$. Thus the tracer particles are dynamically identical to the host particles (for more details, see Refs. $[7,15])$.

Fourth, all measured ISFs are based on the full ensemble of phases, $\mathbf{q} \cdot \Delta \mathbf{r}[$ Eq. (1)]. This is ensured by a sufficiently large scattering volume and, for samples near the GT where fluctuations are extremely sluggish, by rotation or translation of the sample [7,22]. In this ensemble the scattered light field is a complex Gaussian variable with zero mean [23]. The criterion for this, satisfied in these experiments, is that the normalized mean-squared amplitude of the intensity fluctuations of the scattered light is 2, after allowing for the effects of multiple scattering and spatial integration over the detector area [24].

Fifth, the self-ISFs discussed here were all made at the lowest wave vector, $q=1.3 / R$, accessible with the configuration of the two-color, multiple scattering suppression, spectrometer [24]. Conveniently, there are no detectable differences between the self-ISF measured at this wave vector and the Gaussian limit [Eq. (3)], i.e., $F_{\mathrm{s}}(q R=1.3, \tau)$ $\cong F_{\mathrm{s}}^{(\mathrm{G})}(q R=1.3, \tau)[7]$.

\section{RESULTS AND DISCUSSION}

Distances are expressed here in units of the particle radius $R$ and delay times in units of the Brownian characteristic time, $\tau_{\mathrm{b}}=R^{2} /\left(6 D_{\mathrm{o}}\right)(=0.021 \mathrm{~s})$. Thus $\tau_{\mathrm{b}}$ is the time interval in which a particle in an ideal dilute suspension executes an RMS displacement equal to its radius.

Typical MSDs are shown in Fig. 1 for several values of $\phi$. In this log-log presentation straight lines of unit slope indicate diffusion. The average interval $\tau_{\mathrm{c}}$ between particle en- 


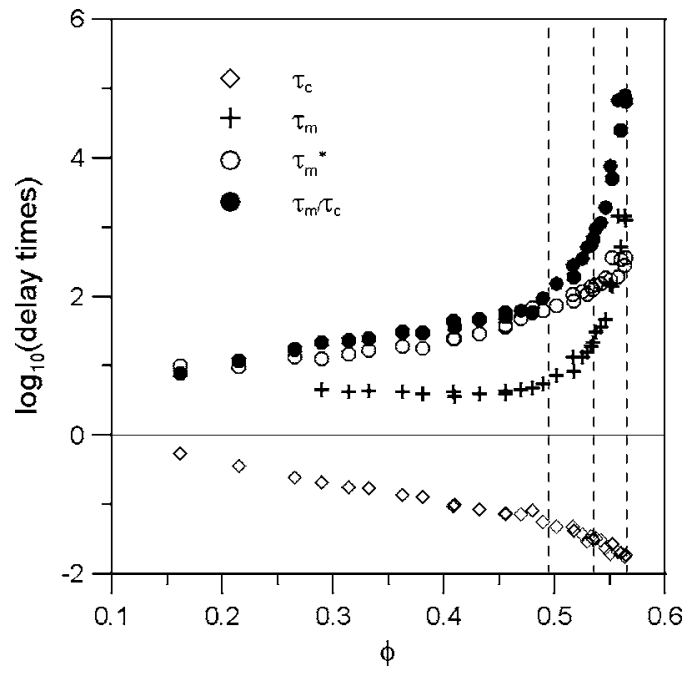

FIG. 2. Characteristic delay times discussed in the text: $\tau_{\mathrm{m}}$ is the time of maximum stretching; $\tau_{\mathrm{c}}$ is the average interval between particle encounters; $\tau_{\mathrm{m}}^{*}$ is the interval where $N(q, \tau)[$ Eq. (7)] has a maximum. The vertical dashed lines, shown in all figures where $\phi$ is the abscissa, are located at the observed freezing, melting, and glass transition volume fractions, $\phi_{\mathrm{f}}=0.494, \phi_{\mathrm{m}}=0.535$, and $\phi_{\mathrm{g}}=0.565$.

counters, defined in Sec. II, is indicated and shown as a function of $\phi$ in Fig. 2, along with other quantities to be discussed later. The short-time self-diffusion coefficients $D_{\text {s }}$ [Eq. (4)], estimated from the intercepts of the best fitting lines of unit slope to the data in the window $\tau<\tau_{\mathrm{c}}$, are shown in Fig. 3 along with results from previous experiments $[7,15,25]$. We attribute differences between the various estimates of $D_{\mathrm{s}}$ to experimental errors.

Figure 4 shows examples of the ISF, $F_{\mathrm{S}}(q, \tau)$, the thermal mode, $T(q, \tau)=\exp \left(-D_{\mathrm{s}} q^{2} \tau\right)$, and the difference, $N(q, \tau)$, defined in Sec. II, for the colloidal fluid in thermodynamic equilibrium $\phi<\phi_{\mathrm{f}}$ [Figs. 4(a) and (b)]; the nonequilibrium fluid in the coexistence region, $\phi_{\mathrm{f}}<\phi<\phi_{\mathrm{m}}$ [Fig. 4(c)]; and

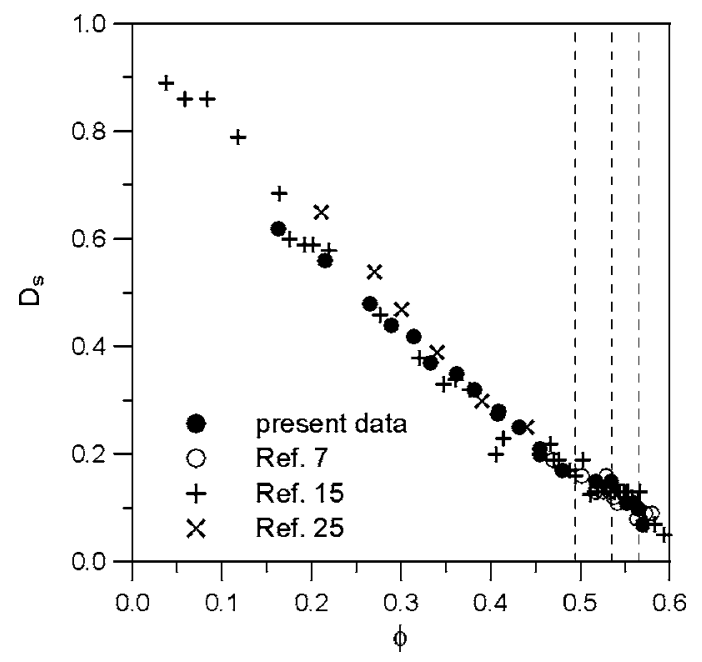

FIG. 3. Short-time self-diffusion coefficients $D_{\mathrm{s}}$ obtained from present and previous experiments. the nonequilibrium fluid above the melting value, $\phi>\phi_{\mathrm{m}}$ [Fig. 4(d)]. The noise and change in spacing of the data around $\log \tau=2$, seen for the highest volume fraction, is the region where the results of two different correlators have been joined (see Ref. [7] for details). The delay time $\tau_{\mathrm{m}}^{*}$ where $N(q, \tau)$ has a maximum, is shown against the volume fraction in Fig. 2.

Obviously, once $T(q, \tau)$ has decayed to zero $N(q, \tau)$ coincides with $F_{\mathrm{s}}(q, \tau)$. The exponential approximation to the subsequent decay of $F_{\mathrm{s}}(q, \tau)$ is obtained by translating $T(q, \tau)$ along the $\log \tau$ axis until the two coincide (see Fig. $4)$. From this translation the ratio $D_{1} / D_{\mathrm{s}}$ of the long and short time self-diffusion coefficients is obtained. As is evident from Fig. 4(d) this procedure may overestimate the value of $D_{1} / D_{\mathrm{s}}$ when $\phi$ is close to $\phi_{\mathrm{g}}$ and the complete decay of the ISF to the noise floor is no longer captured in the experimental window. Aside from this ambiguity this procedure estimates the linear transport coefficient $D_{1}$ that characterizes the Markovian approximation to the slowest detectable tagged particle density fluctuations. The coefficients $D_{1}$ are shown in Fig. 5 in a semilog presentation along with estimates from other experiments $[7,15]$.

Figure 6 shows the amplitudes $T\left(q, \tau_{\mathrm{m}}^{*}\right)$ and $N\left(q, \tau_{\mathrm{m}}^{*}\right)$ of the thermal and non-Markovian modes at $\tau_{\mathrm{m}}^{*} . T\left(q, \tau_{\mathrm{m}}^{*}\right)$ decreases monotonically with volume fraction and falls below the noise floor $(\approx 0.01)$ at $\phi \approx \phi_{\mathrm{m}}$. Thus once the melting volume fraction is exceeded it appears that structural relaxation can no longer be accounted for in terms of (thermally driven) diffusive encounters alone. However, aside from this inference, analysis of the data so far reveals no clear qualitative difference between the equilibrium and undercooled colloidal fluids.

We now turn to the second method, described in Sec. II, to separating fast and slow contributions to the ISF. This requires the point of maximum stretching of the MSD which, for $\phi>0.3$, can be determined directly by application of Eq. (8) to the data. The delay times $\tau_{\mathrm{m}}$, shown in Fig. 2, show no systematic variation with $\phi$ up to approximately $\phi_{\mathrm{f}}$. For larger $\phi, \tau_{\mathrm{m}}$ increases, seemingly without limit on approaching $\phi_{\mathrm{g}}$.

For $\phi<0.3$ deviations from diffusion are apparent but they are too small for the point of inflection, in the double logarithm plot of the MSD vs $\tau_{\mathrm{m}}$, to be discernable from the experimental noise. In these cases we set $\tau_{\mathrm{m}}$ to the average value $\left(\left\langle\tau_{\mathrm{m}}\right\rangle=4\right)$ found for $0.3<\phi<\phi_{\mathrm{f}}$. The index $\nu$ is then obtained from the logarithmic derivative of the MSD at $\tau=\left\langle\tau_{\mathrm{m}}\right\rangle$. The results are shown for all $\phi$ in Fig. 7 . Note that $\nu$ decreases monotonically to zero as $\phi_{\mathrm{g}}$ is approached and passes through approximately $\frac{1}{2}$ and $\frac{1}{3}$ at $\phi_{\mathrm{f}}$ and $\phi_{\mathrm{m}}$, respectively. These particular values of the exponent emerge in the asymptotic growth of the MSD in a number of model systems, such as single file diffusion [26], the Lorentz gas [27], and random walks in the presence of traps [28], all of which suggest that the movement exposed in the undercooled fluid at delay time $\tau_{\mathrm{m}}$ is movement confined to one dimension. We defer a detailed analysis of the data in terms of these models to a future publication.

Given $\tau_{\mathrm{m}}$, the amplitude $A(q)$ and the quantity $P(q, \tau)$, defined by Eqs. (9) and (10), can be calculated. In Fig. 6 one 

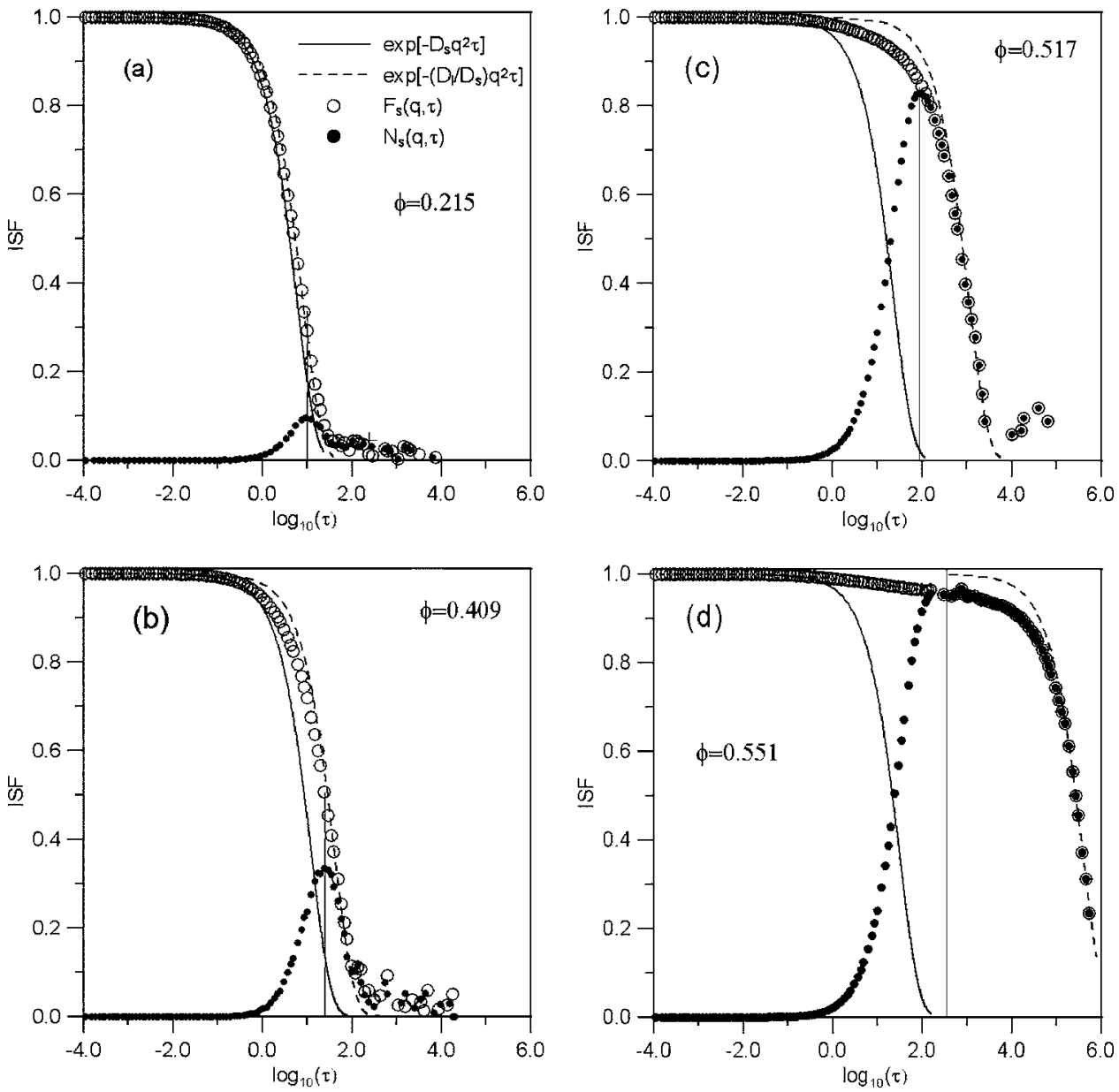

FIG. 4. Self-ISF, $F_{\mathrm{s}}(q, \tau)$, the thermal mode, $T(q, \tau)=\exp \left[-D_{\mathrm{s}} q^{2} \tau\right]$, and the non-Markovian mode, $N(q, \tau)$ for several volume fractions. The vertical lines mark the delay times $\tau_{\mathrm{m}}^{*}$ where $N(q, \tau)$ is a maximum.

sees that $A(q)$ increases monotonically up to $\phi_{\mathrm{f}}$ and then remains constant.

$P(q, \tau)$ is shown in Fig. 8 for cases that typify behavior of the colloidal fluid in the thermodynamically stable region, in

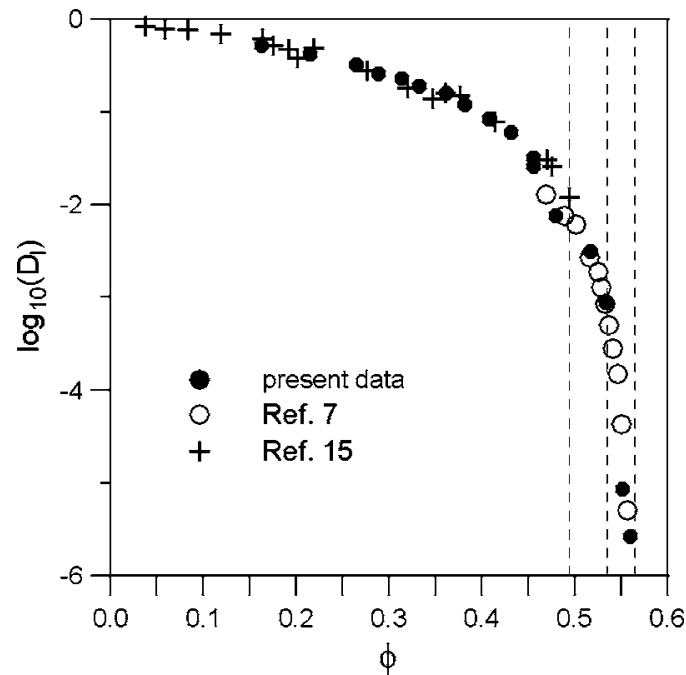

FIG. 5. Long-time self-diffusion coefficients $D_{1}$ obtained from present and previous experiments. the coexistence region and above the melting volume fraction. The behavior of $P(q, \tau)$ in the immediate vicinity of the cusp $\left[P\left(q, \tau_{\mathrm{m}}\right)=0\right]$ is very sensitive to experimental uncer-

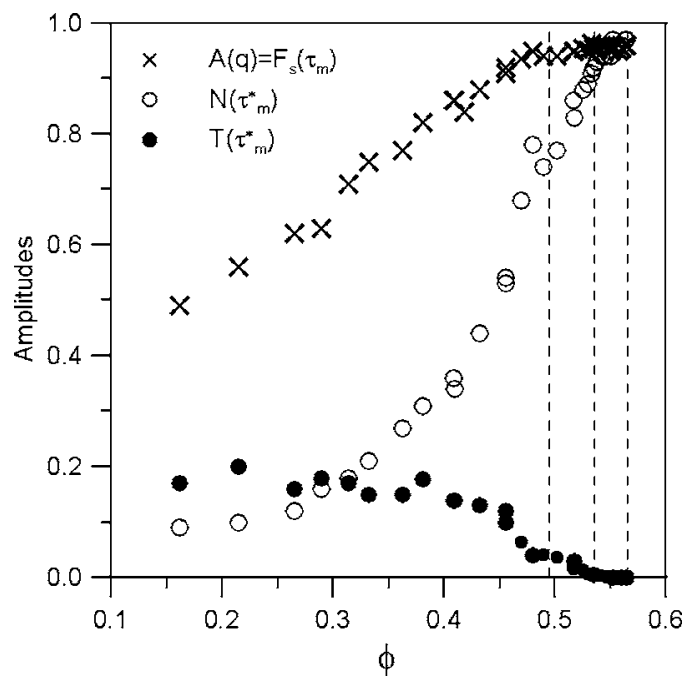

FIG. 6. The amplitudes $T\left(q, \tau_{\mathrm{m}}^{*}\right)$ and $N\left(q, \tau_{\mathrm{m}}^{*}\right)$ of the thermal and non-Markovian modes at $\tau_{\mathrm{m}}^{*}$ [defined by Eqs. (6) and (7)], and the amplitude $A(q)$ defined by Eq. (9). See text for details. 


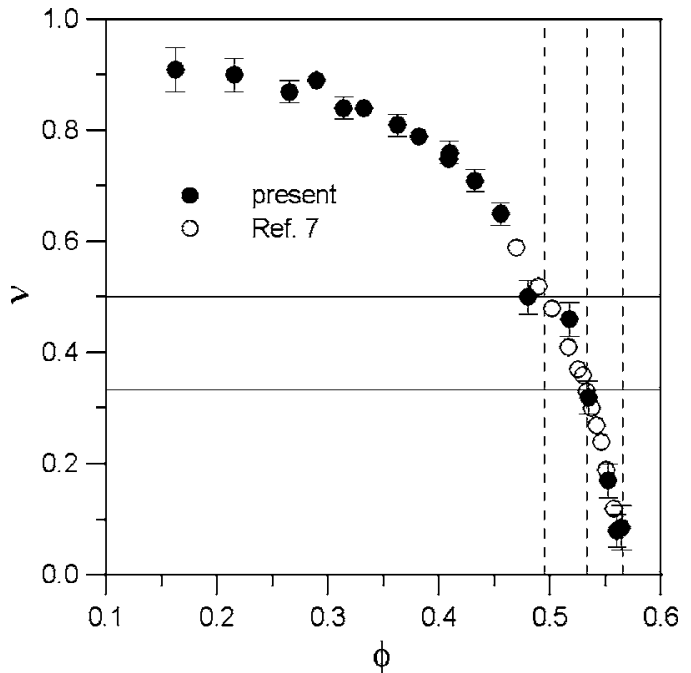

FIG. 7. Stretching index $\nu$ defined by Eq. (8), as a function of volume fraction. The horizontal lines represent the value of $\nu$ as it crosses freezing $\left(\nu=\frac{1}{2}\right)$ and melting $\left(\nu=\frac{1}{3}\right)$.

tainty. Away from this point the results are more stable and we are able to identify qualitative differences in the decay of both the fast and slow contributions in different regions of the phase diagram.

We begin with the contribution $P\left(q, \tau<\tau_{\mathrm{m}}\right)$ of the fast fluctuations which, as seen in Fig. 9(a), shows no systematic variation with $\phi$ for $\phi<\phi_{\mathrm{f}}$. Fitting the data to the function,

$$
P\left(q, \tau<\tau_{\mathrm{m}}\right)=\exp \left[-\left(\tau / \tau_{\mathrm{e}}\right)^{\beta}\right],
$$

treating both $\tau_{\mathrm{e}}$ and $\beta$ as free parameters yields $\beta$ $=1.00 \pm 0.05$ and the characteristic decay times $\tau_{\mathrm{e}}$, shown in Fig. 10(a). The ratio $\tau_{\mathrm{m}} / \tau_{\mathrm{e}}(\cong 2)$ [Fig. 10(b)] shows no variation with $\phi$. In other words, for the equilibrium suspension the fast process, defined by Eq. (10), can be described by an exponential function of delay time that shows no systematic variation with volume fraction.

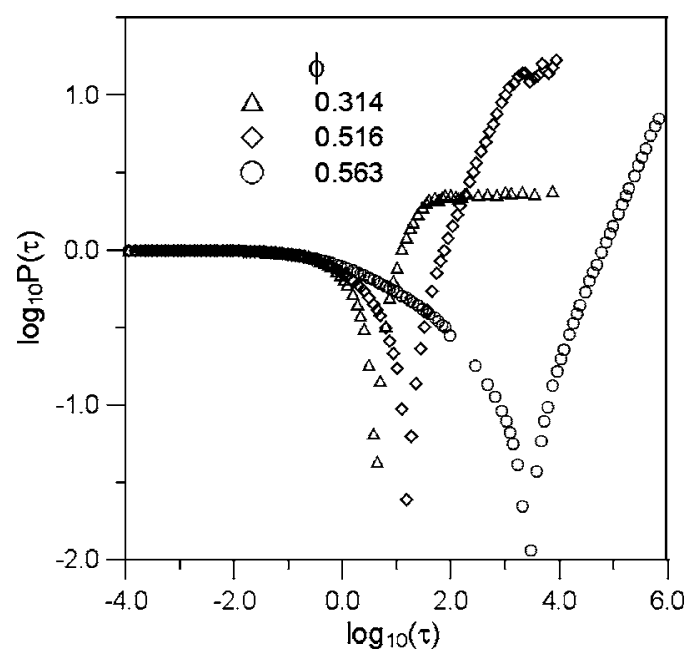

FIG. 8. The quantity, $P(q, \tau)$, defined by Eq. (10) for the volume fractions indicated.
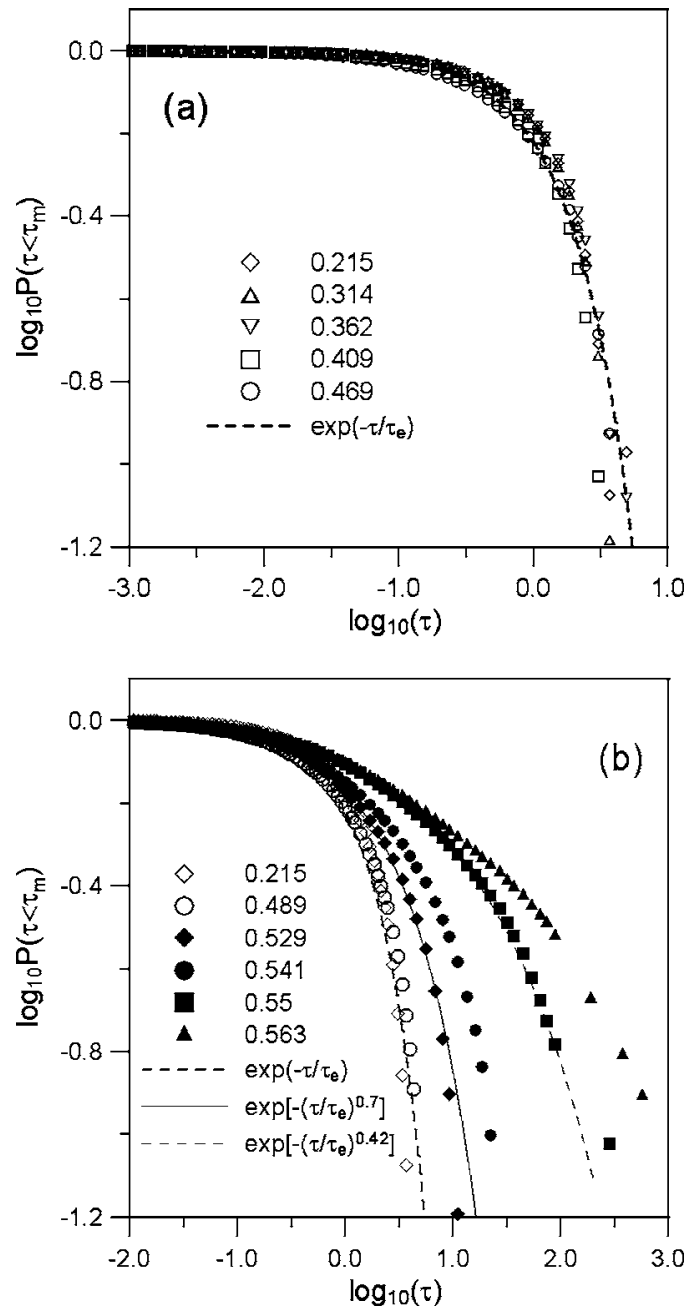

FIG. 9. The fast decay, $P\left(q, \tau<\tau_{\mathrm{m}}\right)$, of the self-ISF at volume fractions indicated. (a) $\phi<\phi_{\mathrm{f}}$ : The dashed curve is Eq. (12) with $\beta=1$ and $\log \tau_{\mathrm{e}}=0.22$. (b) $\phi>\phi_{\mathrm{f}}$ : The curves are Eq. (12) for various values of $\beta$. Note the difference in scale of the abscissas of (a) and (b).

As seen in Fig. 9(b), when the volume fraction exceeds the freezing value, $\phi>\phi_{\mathrm{f}}$, the decay of the fast fluctuations becomes increasingly stretched. For these cases $P\left(q, \tau<\tau_{\mathrm{m}}\right)$ has been fitted to the stretched exponential, Eq. (12), with $\tau_{\mathrm{e}}$ and $\beta$ as free parameters. The values obtained for these are shown in Figs. 10 and 11, respectively. The quantity $\tau_{\mathrm{s}}$ is discussed below. The expanded scale of Fig. 10 (b) gives a clearer view of the noise on the various characteristic times. Despite this there appears to be no systematic dependence of the ratio $\tau_{\mathrm{e}} / \tau_{\mathrm{m}}$ on $\phi$. The best fitting straight line to the values of $\beta$ gives $\beta\left(\phi_{\mathrm{f}}\right) \approx 1$ and $\beta\left(\phi_{\mathrm{g}}\right)$ $\approx 1 / 3$. Thus the fast process in the undercooled colloidal fluid is able to be described by the stretched exponential with the stretching exponent $\beta$ decreasing from 1 at $\phi_{\mathrm{f}}$ to approximately $\frac{1}{3}$ at $\phi_{\mathrm{g}}$.

Next we examine the slow component of the fluctuations, $P\left(q, \tau>\tau_{\mathrm{m}}\right)$. Here we are able to identify three qualitatively different behaviors. In the first, illustrated in Fig. 12(a), there is consistency between $P\left(q, \tau>\tau_{\mathrm{m}}\right)$ and that calculated from 

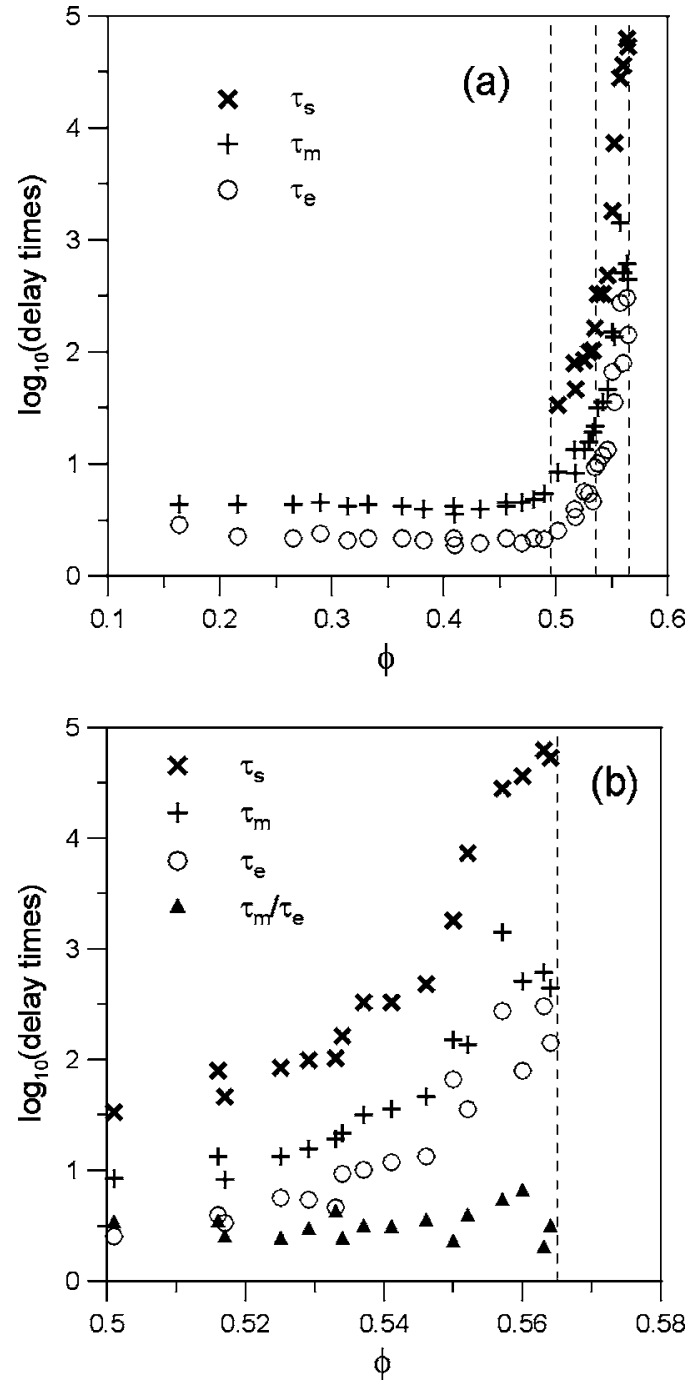

FIG. 10. Characteristic times and scaling times: (a) shows $\tau_{\mathrm{m}}$, the time of maximum stretching; $\tau_{\mathrm{s}}$ [defined by Eq. (14)]; $\tau_{\mathrm{e}}[$ defined by Eq. (12)]. (b) shows these quantities on an expanded scale and also shows the ratio $\tau_{\mathrm{m}} / \tau_{\mathrm{e}}$.

Eq. (10) with $A(q)$ from Fig. 6 and $F_{\mathrm{s}}(q, \tau)=\exp \left(-D_{1} q^{2} \tau\right)$, where $D_{1}$ is the long-time self-diffusion constant shown in Fig. 5. This consistency is found within experimental error for $\phi$ below approximately 0.35 . We express this consistency with the generalized form,

$$
P\left(q, \tau>\tau_{\mathrm{m}}\right)=\frac{\exp \left[-\left(D_{1} q^{2} \tau\right)^{\gamma}\right]-A(q)}{1-A(q)},
$$

which allows for possible stretching of the slow process. Thus for $\phi<0.35$ Eq. (13) describes the data with $\gamma=1$.

The second type of behavior is shown in Fig. 12(b) for $0.35<\phi<\phi_{\mathrm{f}}$. Clearly Eq. (13) with $\gamma=1$ no longer describes the data. In these cases much better agreement is achieved by allowing $\gamma$ to be less than 1. As can be seen in Fig. 13, the value of $\gamma$ that give the best fit to the data shows a small but systematic decrease with $\phi$.

Third, when the volume fraction exceeds the freezing value and one presses deeper into the undercooled region, it

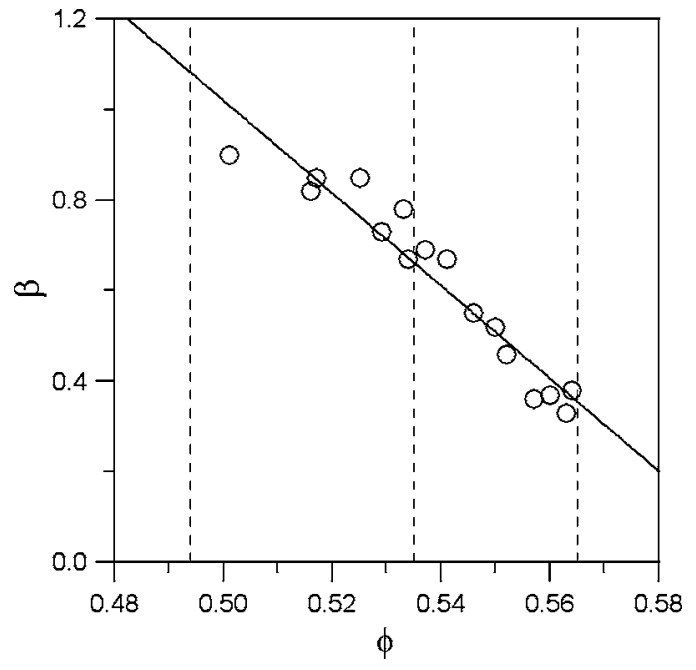

FIG. 11. The exponent $\beta$ defined by Eq. (12) as a function of volume fraction. The line of best fit is also shown.

becomes increasingly difficult to describe the data with Eq. (13). As seen in Fig. 12(c), in these cases a power law,

$$
P\left(q, \tau>\tau_{\mathrm{m}}\right)=\left(\tau / \tau_{\mathrm{s}}\right)^{\lambda},
$$

emerges as the better description of the data. For consistency Eq. (14) has been fitted to the data in the range $-0.5 \leqslant \log P\left(q, \tau>\tau_{\mathrm{m}}\right) \leqslant 1.0$. Below the lower limit $P(q, \tau$ $\left.>\tau_{\mathrm{m}}\right)$ is very sensitive to $\tau_{\mathrm{m}}$, whereas above the upper limit it approaches the experimental noise floor. The resulting scaling times $\tau_{\mathrm{s}}$ and exponents $\lambda$ are shown in Figs. 10 and 13 , respectively.

To determine whether there is any connection between the scaling time $\tau_{\mathrm{s}}$ and the decay time $\left(6 / q^{2} D_{1}\right)$ that characterizes (long-time) diffusion, the product $\left(q^{2} D_{1} / 6\right) \tau_{\mathrm{s}}$ is also shown in Fig. 13. Given the experimental noise we cannot realistically discern any systematic variation of $\left(q^{2} D_{1} / 6\right) \tau_{\mathrm{s}}$ from its average value of 0.04 . Thus barring any variation that may be hidden by the experimental noise, we infer that the process that emerges at $\phi_{\mathrm{f}}$ is universal in that the exponent $\lambda$ of the power law is independent of $\phi$ and that $\tau_{\mathrm{s}}$ scales with the inverse of the long-time diffusion coefficient.

\section{SUMMARY AND CONCLUSION}

We have examined the time correlation function of the Gaussian component of the tagged particle density fluctuations of a suspension of hard-sphere particles, with the purpose of exposing qualitative differences between equilibrium $\left(\phi<\phi_{\mathrm{f}}\right)$ and nonequilibrium $\left(\phi>\phi_{\mathrm{f}}\right)$ conditions. To this end two methods have been employed to separate the ISF into slow and fast contributions. The two methods give two different (dimensionless) quantities, $\tau_{\mathrm{m}}$ and $\tau_{\mathrm{m}}^{*}$ (Fig. 2), that characterize the non-Markovian process in the suspension. Determination of $\tau_{\mathrm{m}}^{*}$, in the first method illustrated in Fig. 4, accounts for this process by accumulation of uncorrelated (diffusive) encounters. No account is taken of the memory of the suspending liquid. Determination of $\tau_{\mathrm{m}}$ in the second 

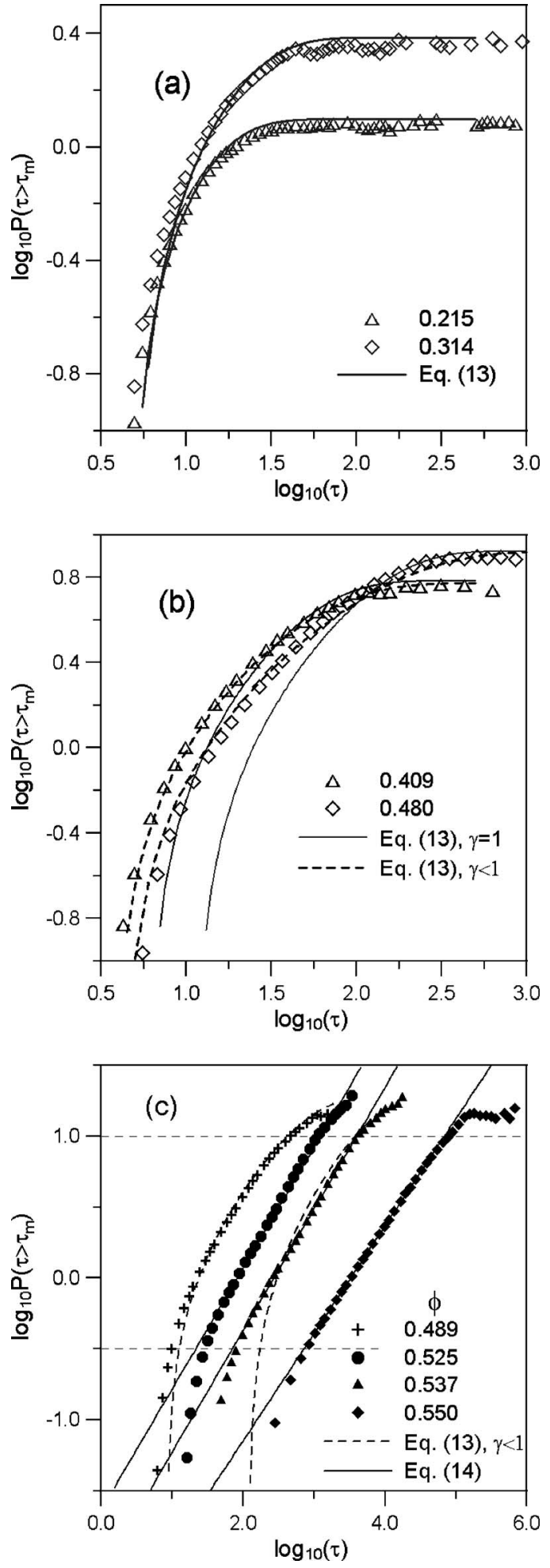

FIG. 12. Slow decay, $P\left(q, \tau>\tau_{\mathrm{m}}\right)$, of the self-ISF at the representative volume fractions indicated. (a) $\phi<0.35$, (b) $0.35<\phi$ $<\phi_{\mathrm{f}}$, and (c) $\phi>\phi_{\mathrm{f}}$. In (a) and (b) the solid curves represent the best fits to the data with Eq. (13) and $\gamma=1$. In (b) the dashed curves represent the best fit to the data with Eq. (13) and $\gamma<1$. The horizontal lines in (c) mark the range over which Eq. (14), shown by the solid lines, has been fitted to the data. For comparison, the dashed curve in (c) represents the best fit with Eq. (13). The fit parameters are shown in Fig. 13.

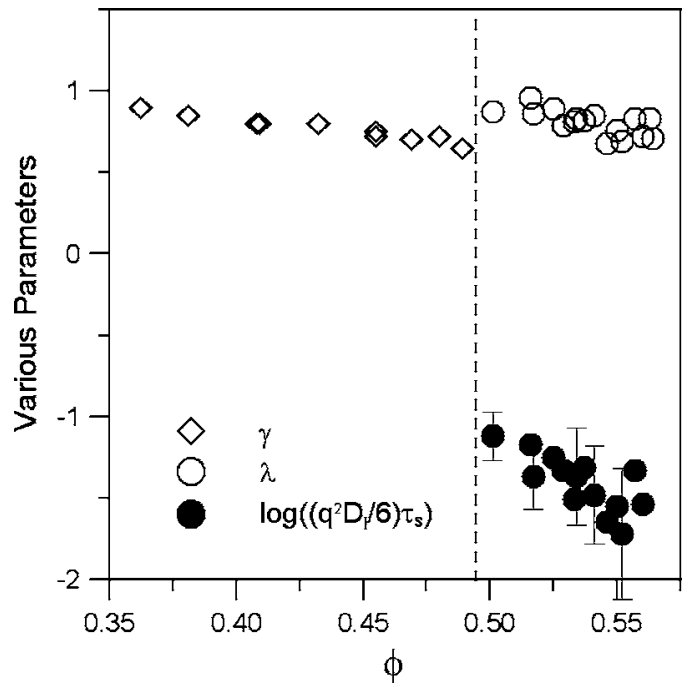

FIG. 13. Shows the parameters $\gamma$ [defined in Eq. (13)], $\lambda[$ defined in Eq. (14)], and the quantity $\log \left[\left(q^{2} D_{1} / 6\right) \tau_{\mathrm{s}}\right]$ as function of volume fraction.

method makes no assumption. The ratio $\tau_{\mathrm{m}} / \tau_{\mathrm{c}}$, also plotted in Fig. 2, gives the number of particle encounters accumulated during $\tau_{\mathrm{m}}$. One sees for the equilibrium colloidal fluid $\left(\phi<\phi_{\mathrm{f}}\right)$ that $\tau_{\mathrm{m}}^{*}$ and $\tau_{\mathrm{m}} / \tau_{\mathrm{c}}$ coincide within experimental uncertainty. Thus structural relaxation, characterized by the interval $\tau_{\mathrm{m}}$, can also be expressed by $\tau_{\mathrm{m}} / \tau_{\mathrm{c}}$ statistically identical diffusive encounters.

For the undercooled colloidal fluid $\left(\phi>\phi_{\mathrm{f}}\right)$ the observation that $\tau_{\mathrm{m}} / \tau_{\mathrm{c}}$ exceeds $\tau_{\mathrm{m}}^{*}$ suggests another source of memory has come into play. Hence, by the reasoning above, structural relaxation can no longer be described entirely in terms of memoryless encounters. In this there is the suggestion of a possible change in the relaxation scenario when the volume fraction of the suspension passes through the freezing value.

The different relaxation scenarios of the equilibrium and undercooled colloidal fluids are exposed explicitly when separating fast fluctuations, defined by those accumulated in the time interval (" 0, , $\left.\tau_{\mathrm{m}}\right)$, from slow fluctuations, defined by those accumulated in the interval $\left(\tau_{\mathrm{m}}\right.$, “ $\infty$ "). This separation reveals the following.

For the colloidal fluid in thermodynamic equilibrium $\left(\phi<\phi_{\mathrm{f}}\right)$ the autocorrelation function of the fast fluctuations decays exponentially with a decay time that is independent of $\phi$, i.e., the fast process is a universal Markovian process. The slow process decays by an exponential $(\phi<0.35)$ or stretched exponential $\left(0.35<\phi<\phi_{\mathrm{f}}\right)$ function of delay time. The characteristic decay time is set by the long-time selfdiffusion coefficient.

For the undercooled colloidal fluid $\left(\phi>\phi_{\mathrm{f}}\right)$ the fast fluctuations decay by a stretched exponential function [Eq. (12)]. The stretching exponent decreases from unity, at $\phi_{\mathrm{f}}$, to about $\frac{1}{3}$ at $\phi_{\mathrm{g}}$, and the characteristic decay interval grows without apparent limit when approaching the volume fraction $\phi_{\mathrm{g}}$ of the glass transition. The slow process follows a power law whose index is independent of $\phi$.

In essence we find that for volume fractions less than about $\phi \approx 0.35$ both fast and slow processes are Markovian, 
or memoryless. At $\phi \approx 0.35$ memory enters the slow process. At $\phi_{\mathrm{f}}$ memory enters the fast process and the slow process becomes scale free. These qualitative changes indicate an awareness on the part of the hard-sphere colloidal fluid of its traversal of not just $\phi_{\mathrm{f}}$ but also $\phi \approx 0.35$.

\section{ACKNOWLEDGMENTS}

The authors would like to thank Peter Daivis, Jan Dhont, Matthias Fuchs, Peter Pusey, Salvy Russo, and Ian Snook for helpful discussions, and for comments on previous versions of this manuscript.
[1] P. G. Debenedetti, Metastable Liquids, Concepts and Principles (Princeton University Press, Princeton, NJ, 1996).

[2] W. C. K. Poon and P. N. Pusey, in Observation, Prediction and Simulation of Phase Transitions in Complex Fluids, edited by M. Baus et al. (Kluwer Academic Publishers, Amsterdam, 1995), p. 3; D. W. Oxtoby, Nature (London) 413, 694 (2001); V. J. Anderson and H. N. W. Lekkerkerker, ibid. 416, 811 (2002).

[3] P. N. Pusey and W. van Megen, Nature (London) 362, 340 (1986).

[4] W. G. Hoover and F. H. Ree, J. Chem. Phys. 49, 3609 (1969).

[5] S. I. Henderson and W. van Megen, Phys. Rev. Lett. 80, 877 (1998).

[6] W. van Megen, Transp. Theory Stat. Phys. 24, 1017 (1995).

[7] W. van Megen, T. C. Mortensen, S. R. Williams, and J. Müller, Phys. Rev. E 58, 6073 (1998).

[8] J. Frenkel, Kinetic Theory of Liquids (Oxford University Press, Oxford, 1946).

[9] J. C. Maxwell, Philos. Trans. R. Soc. London 157, 49 (1867).

[10] W. Götze, in Liquids, Freezing and the Glass Transition, edited by J. P. Hansen, D. Levesque, and J. Zinn-Justin (NorthHolland, Amsterdam, 1991), p. 287.

[11] W. van Megen and S. M. Underwood, Phys. Rev. E 49, 4206 (1994).

[12] W. Götze, J. Phys.: Condens. Matter 11, A1 (1999).

[13] W. van Megen, J. Phys.: Condens. Matter 14, 7699 (2002).

[14] B. J. Berne and R. Pecora, Dynamic Light Scattering (John
Wiley, New York, 1976); Dynamic Light Scattering, The Method and Some Applications, edited by W. Brown (Clarendon Press, Oxford, 1993).

[15] W. van Megen and S. M. Underwood, J. Chem. Phys. 91, 552 (1989); Langmuir 6, 35 (1990).

[16] B. R. A. Nijboer and A. Rahman, Physica (Amsterdam) 32, 415 (1966).

[17] P. N. Pusey, in Liquids, Freezing and the Glass Transition (Ref. [9]), p. 763.

[18] D. L. Ermak and J. A. McCammon, J. Chem. Phys. 69, 1352 (1978).

[19] J. M. Deutsch and I. Oppenheim, J. Chem. Phys. 54, 3547 (1971); T. J. Murphy and J. L. Aguirre, ibid. 57, 2098 (1972).

[20] G. Bryant et al., Phys. Rev. E 66, 060501(R) (2002).

[21] P. G Bolhuis and D. A. Kofke, Phys. Rev. E 54, 634 (1996).

[22] W. van Megen and S. M. Underwood, Phys. Rev. E 47, 248 (1993).

[23] P. N. Pusey and W. van Megen, Physica A 157, 705 (1989).

[24] P. N. Segrè, et al., J. Mod. Opt. 42, 1929 (1995).

[25] P. N. Pusey and W. van Megen, J. Phys. (Paris) 44, 285 (1983).

[26] T. E. Harris, J. Appl. Probab. 2, 323 (1965); C. Lutz, M. Kollmann, and C. Bechinger, Phys. Rev. Lett. 93, 026001 (2004).

[27] M. H. Ernst and A. Weyland, Phys. Lett. 34A, 39 (1971).

[28] P. Grassberger and I. Procaccia, J. Chem. Phys. 77, 6281 (1982); R. F. Kayser and J. B. Hubbard, Phys. Rev. Lett. 51, 79 (1983). 